\title{
ARQUITECTURA HINCHABLE CONTEMPORÁNEA LA FUNCIONALIDAD DE LO EFÍMERO
}

\section{CONTEMPORARY INFLATABLE ARCHITECTURE THE FUNCTIONALITY OF THE EPHEMERAL}

\author{
Carmen Flores Hurtado \\ (Universidad Complutense de Madrid, España) \\ carmf101@ucm.es
}

Recibido: 25 febrero 2020 Aceptado: 08 marzo 2020

\begin{abstract}
Resumen: La arquitectura como disciplina ya no es lo que era; con los cambios artísticos experimentados desde la segunda mitad del siglo XX, todo ha mutado hacia nuevos paradigmas en los que se conciben formas novedosas de hacer y construir. Ejemplo de ello es la aparición de la arquitectura hinchable en los años 60 con proyectos como la Instant City de Ibiza por parte del arquitecto José Miguel de Prada Poole. En este artículo se verán algunos proyectos actuales que siguen reinventando la manera de construir.
\end{abstract}

Palabras clave: Arquitectura efímera, Arquitectura hinchable, Funcionalidad, Espacio, Aire.

\begin{abstract}
Architecture as a discipline is no longer what it was; With the artistic changes experienced since the second half of the 20th century, everything has mutated into new paradigms in which novel ways of doing and building are conceived. An example of this is the appearance of inflatable architecture in the 1960s with projects such as the Instant City of Ibiza by the architect José Miguel de Prada Poole. In this article we will see some current projects that continue to reinvent the way of building.
\end{abstract}

Keywords: Ephemeral architecture, Inflatable architecture, Funcionality, Space, Air. 


\section{INTRODUCCIÓN}

Con los conocidos cambios que el panorama artístico comenzó a experimentar a mediados del siglo XX, nacieron novedosas formas de expresión que aún hoy en día siguen desarrollándose. La dimensión de nuestros espacios públicos ha sido y es muy importante para el desarrollo de estas expresiones, por ello ambos van creciendo y cambiando en una misma dirección. El arte efímero es clave para entender los procesos que está experimentando el panorama artístico actual, pues consigue una comunicación con el espectador que resulta mucho más complicada con las prácticas artísticas tradicionales.

Ante la dificultad de afrontar todos los procesos que se dan en la actualidad, surgen diferentes maneras de hacer arte que van adaptándose a los cambios que solicita la sociedad. Las disciplinas artísticas van entendiendo cada vez más al espectador y al ciudadano, y haciéndose eco de sus necesidades; por ello muta, cambia y se desarrollan otras nuevas maneras de crear.

En el campo de la arquitectura, desde los años 60 del siglo XX se han ido experimentando notables cambios, con la aparición de arquitectos que sintieron la necesidad de romper con lo tradicional y con lo impuesto, y comenzaron a idear formas nuevas de construcción para solventar las necesidades de una sociedad cambiante.

En el presente artículo se abordarán algunas cuestiones fundamentales para entender el desarrollo de la arquitectura efímera hinchable, haciendo hincapié en algunos ejemplos de artistas contemporáneos que vienen desarrollando sus trabajos en ciudades españolas y que han salido a la calle dispuestos a transformar la noción de nuestros espacios.

El objetivo principal de este artículo es el de poner en conocimiento formas de expresión novedosas y funcionales que se desarrollan dentro del arte efímero, además de ahondar en una disciplina poco estudiada como es la arquitectura hinchable, a través de las figuras de los arquitectos y colectivos más señeros en el ámbito nacional e internacional. 


\section{ARQUITECTURA HINCHABLE: CONSTRUIR SUEÑOS CON AIRE}

La ciudad cambia a medida que cambian sus ciudadanos y las necesidades de éstos. Es un hecho que, en los últimos años, la concepción del espacio público ha mutado por completo y es, en parte, debido a la presencia de las expresiones artísticas en él. La arquitectura también se ha ido adaptando a todos estos cambios y ha ido evolucionando hacia nuevas maneras de expresar.

La primera mitad del siglo XX fue de la mano del Movimiento Moderno ${ }^{1}$, una corriente que llegó como una revolución, empleando nuevos materiales y formas de construir. Pero es cierto que no fue hasta pasados los años 50 cuando se rompe de forma radical con todo y hasta con el propio Movimiento Moderno. Así, se da paso a una nueva corriente arquitectónica arriesgada. Surgen una serie de arquitectos en todo el mundo dispuestos a apostar por una nueva visión. Es a partir de los 60 cuando el arte se revoluciona en todas sus vertientes, y los nuevos creadores y pensadores tienen la necesidad de redescubrir y repensar el mundo que les rodea. Es entonces cuando se comienza a experimentar en todos los campos artísticos, produciéndose una apertura de las $\operatorname{artes}^{2}$.

Uno de los aspectos a repensar y redescubrir es la propia ciudad; es por ello que multitud de arquitectos sienten la necesidad de crear algo nuevo y rompedor que se aleje de todo lo racional que había inundado las ciudades en la primera mitad del XX. De esta forma, surge una nueva corriente que es denominada «Arquitectura Experimental», o también «Utópica», «Visionaria» (Blasco, 2017, p. 8), todo ello con el manifiesto del Team $\mathrm{X}^{3}$ como precedente, cuyo principal cuestionamiento estaba dirigido a las ideas de la modernidad ${ }^{4}$.

\footnotetext{
${ }^{1}$ El Movimiento Moderno fue uno de los movimientos artísticos más importantes. Dentro de la arquitectura, se basaba en criterios de funcionalidad y el nuevo uso de materiales como el hormigón y el acero. Buscaba romper con la tradición y renovar las formas compositivas. Entre los artistas más destacados destacan Wlater Gropius o Le Corbusier, entre otros. Alcanzó su máximo esplendor en los años 20 y 30 del siglo XX. Más información en: https://www.hisour.com/es/modern-movement-33608/.

${ }^{2}$ Con «apertura de las artes» se hace alusión al surgimiento y resurgimiento de tantas formas de expresión que se dan a partir de los años 60 .

${ }_{3}$ El Team X fue un grupo de arquitectos que elaboraron un manifiesto titulado Manifiesto de Doorn en 1954 en el que se cuestionan ideas en torno al urbanismo y la arquitectura.

${ }^{4}$ Más información en: https://arquiteorias.blogspot.com/2011/05/el-manifiesto-de-doorn-team-10.html
} 
En este contexto resurge el término de la «Utopía», concepto en que se empiezan a apoyar un gran número de creadores para definir el mundo que quieren crear. Y a raíz de esto comienza a tener importancia la arquitectura hinchable, es decir, la basada en la creación de espacios cuya estructura es sostenida con aire a presión; a simple vista, proyectos utópicos que acaban convirtiéndose en reales. Un tipo de arquitectura manejable, económico y cómodo para ser instalado en los espacios públicos. Estructuras que rompen con lo tradicional, de carácter flexible y atractivas para los espectadores.

Así, en todo el mundo surgen arquitectos y colectivos que comienzan a hacer uso de la arquitectura hinchable, como el caso de Ant Farm ${ }^{5}$ en San Francisco, Archigram ${ }^{6}$ en Londres o $\mathrm{UFO}^{7}$, en Italia. Todos ellos creando estructuras de carácter utópico e impensable pero posibles y realizables, que comenzaban a replantear otras perspectivas en el campo arquitectónico y que, a su vez, atendían a nuevas necesidades de la sociedad, haciendo también uso de la tecnología, que cada vez iba tomando más presencia en este campo.

En España también tuvo su importancia esta nueva arquitectura hinchable y, sobre todo, destaca la figura de José Miguel de Prada Poole (Valladolid, 1938), un arquitecto que construyó obras efímeras con aire. Uno de sus proyectos más conocidos es la Instant City [Fig.1] que se asentó en Ibiza en 1971. Fue una obra de carácter efímero destinada a alojar a los estudiantes asistentes al VII Congreso Internacional de Diseño, convocado por el ICSID ${ }^{8}$. El lugar elegido para instalar esta ciudad instantánea fue la Cala de San Miguel, donde fueron instalados todos los módulos que acabaron constituyendo esta ciudad temporal promovida con la autoconstrucción colaborativa9 ${ }^{9}$ (Prieto, 2013, pp. 27-

\footnotetext{
5 Ant Farm fue un grupo de arquitectos que realizó trabajos experimentales entre los años 60 y 70. Publicaron un manual denominado Inflatocookbook en el que enseñaban a realizar proyectos de arquitectura hinchable. Más información en: https://www.plataformaarquitectura.cl/cl/02-18400/ant-farm.

6 Archigram fue un grupo creado por los arquitectos Peter Cook, David Greene y Mike Webb. Promovieron la creación de estructuras móviles.

7 UFO fue el nombre con el que seis arquitectos italianos denominaron a su grupo anarquista. Realizaron una serie de performances en Florencia haciendo uso de las estructuras hinchables.

${ }^{8}$ Siglas del International Council of Societies of Industrial Design.

9 La ciudad estuvo compuesta de diferentes módulos que se iban conectando entre sí y los cuáles fueron instalados por los propios estudiantes que los iban a ocupar. A cada uno de ellos se le asignó una especie de manual de instrucciones y unas herramientas para poder poner en pie su módulo.
} 
29). Se levantó en dos semanas y durante otras dos se mantuvo en pie, como una utopía hecha realidad, como un sueño construido por aire ${ }^{10}$.

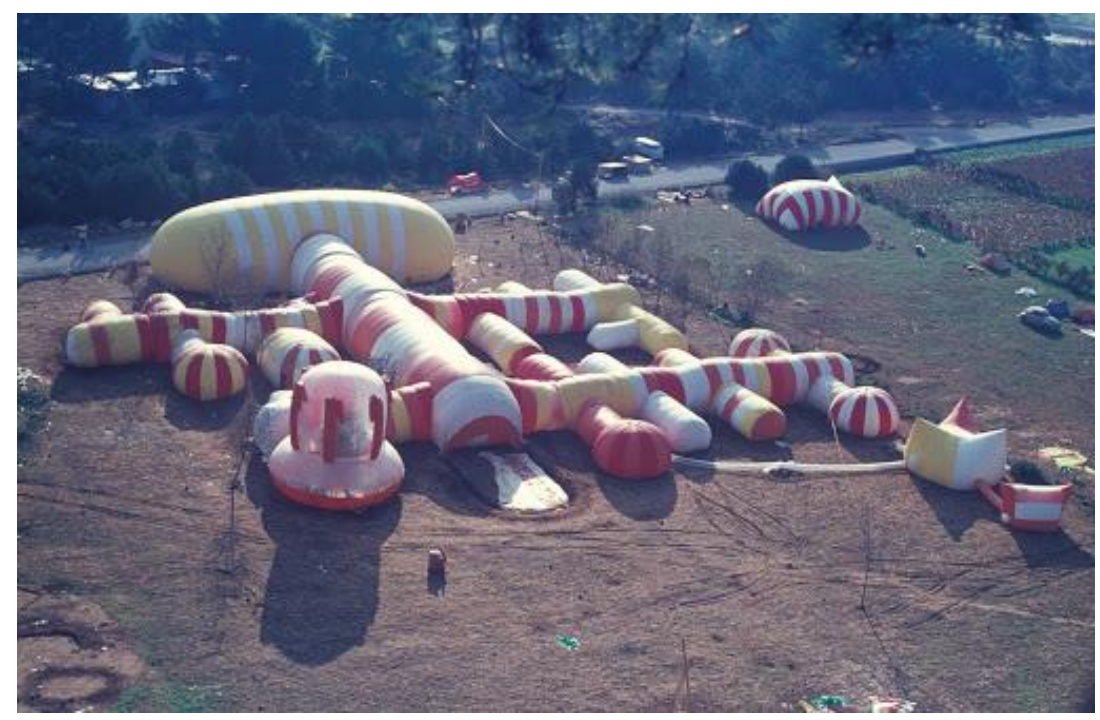

1. Imagen de la Ciudad Instantánea creada por José Miguel de Prada Poole en 1971

\section{ARQUITECTURA HINCHABLE CONTEMPORÁNEA: LOS HABITABLES EFÍMEROS}

En la actualidad, en un mundo cada vez más rápido y cambiante, no es nada descabellado pensar que el arte efímero puede ser la razón de todo; un arte capaz de conmover y revolucionar en un corto lapso de tiempo. Y es que, hoy en día, estamos acostumbrados a vivir experiencias efímeras, con fecha de caducidad. Dentro del panorama artístico, ya quedó lejos la idea del «para siempre», sobre todo en el ámbito arquitectónico, en el cual hace tiempo que la perdurabilidad es un concepto cuestionable a la hora de construir, pues todo depende de para qué y según lo que se ofrezca. Y es que quizá, en muchas ocasiones, es precisamente el componente efímero el que da pie a que muchos proyectos artísticos salgan adelante.

Por todo ello, a día de hoy, la arquitectura efímera desempeña un papel muy señero en nuestra sociedad, ya que es capaz de producir potentes significados y perdurar, aunque no de forma física, sino en nuestras mentes. En la actualidad, la arquitectura es concebida como algo más que una estructura estética y funcional; multitud de arquitectos, como el

10 https://www.jotdown.es/2013/08/construir-castillos-con-el-aire-la-ciudad-instantanea-de-ibiza/. 
citado José Miguel de Prada, realizan proyectos creyendo en el componente social y en el espíritu cambiante de la sociedad, y son muchos otros los que ven en la arquitectura efímera un gran potencial, pues son estructuras medianamente fáciles de construir y que suplen, de forma sencilla y en un corto plazo, una serie de necesidades que difícilmente supliría la arquitectura tradicional de forma tan inmediata.

En todo el mundo es frecuente ver cómo multitud de arquitectos se lanzan a la realización de proyectos efímeros como una vía de escape y una búsqueda de oportunidades dentro de un sector cada vez más inaccesible. En España, debido a la crisis que se vivió a partir de 2008, numerosos profesionales comenzaron a poner su atención en el arte efímero. Y dentro de este mundo de efimeridad, es la arquitectura hinchable una de las formas de creación más experimentadas. En los últimos años se han impulsado los proyectos cuyo protagonista es el aire y esta nueva forma de construir ha comenzado a ser más visible y a instalarse de forma temporal en los espacios públicos de nuestras ciudades.

En los últimos años se han podido ver algunas de estas instalaciones hinchables en diversas ciudades españolas. Es necesario destacar el trabajo del colectivo Plastique Fantastique $^{11}$, cuyo fundador es el arquitecto italiano Marco Canevacci. Este grupo artístico comenzó a hacer uso del plástico por ser un material económico, dando así lugar a estructuras habitables e hinchables de carácter efímero que dotan a los espacios de significados nuevos.

Una de las instalaciones realizadas en España fue la titulada Burbuja Manchega (2007), concebida como un pabellón expositivo móvil en el que se podían realizar talleres y actividades en su interior [2]. Estuvo girando por cinco ciudades diferentes de Castilla La Mancha y aportando nuevos significados a los espacios públicos en que fue instalada.

${ }^{11}$ Web: https://plastique-fantastique.de/ 


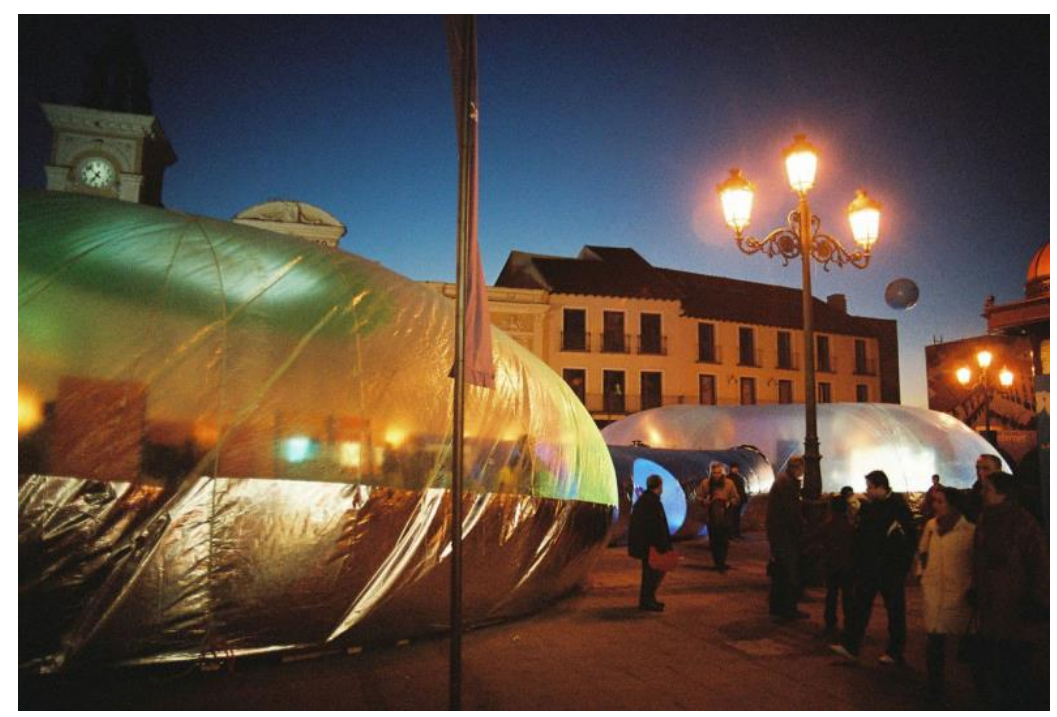

2. Obra de Plastique Fantastique titulada Burbuja Manchega

De igual modo, en 2013 tuvo lugar en el Instituto Europeo de Diseño de Madrid un workshop con el propio Marco Canevacci en el que se instalaron estructuras hinchables efímeras. Sinestesia fue el nombre que se le puso al resultado de este conjunto de instalaciones-performances que buscaban generar reflexiones en torno a la habitabilidad.

En el resto del mundo, Plastique Fantastique ha realizado atractivos proyectos que merecen ser mencionados como el KulturPaLupe (2018), una instalación hinchable realizada en Dresde y construida junto a doce estudiantes de arquitectura. Se propuso como un lugar de intercambio, comunicación y pensamiento.

En la línea de Plastique Fantastique, es necesario hacer alusión a Penique Productions ${ }^{12}$, un colectivo artístico con sede en Barcelona centrado en las instalaciones efímeras. Crean obras únicas y adaptadas a cada espacio con las estructuras hinchables como protagonistas.

Una de sus obras más destacables fue realizada en el municipio de Piera bajo el título La Capella (2009), realizada en la capilla gótica de Sant Sebastià (s. XVI). Se jugó con las luces y las sombras, y una enorme estructura hinchable cubrió todo el interior de

${ }^{12}$ Web: http://peniqueproductions.com/ 
la capilla, transformando por completo la imagen tradicional del lugar y dando paso a una nueva concepción del espacio, mutado también con las tecnologías y luminarias [3].

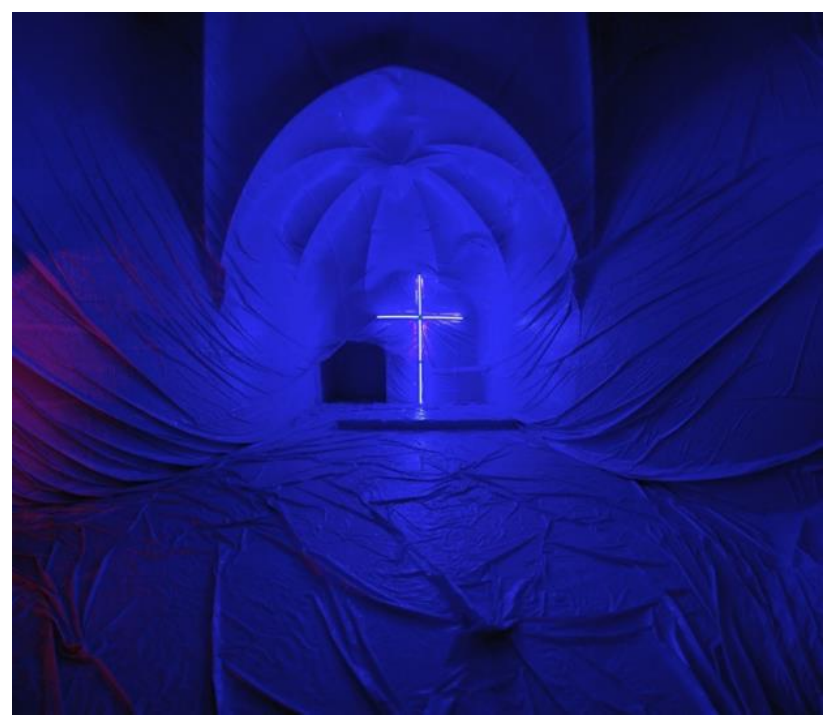

3. Obra La Capella de Penique Productions

En cuanto a proyectos internacionales, es interesante destacar algunas obras como la arquitectura llevada a cabo por Arata Isozaki y Anish Kapoor en 2013. Juntos concibieron una gran sala de conciertos inflable, con el propósito de que ésta recorriera las regiones más afectadas por el terremoto y tsunami que sufrió Japón en 2011. Se tituló Ark Nova [4] y es otro de los ejemplos que se fusionan con el espacio a la perfección, creándose una alternativa habitable.

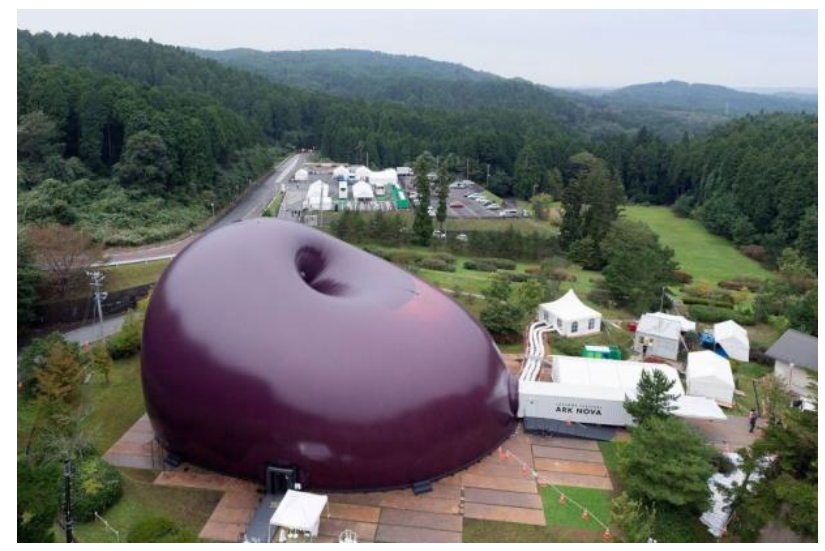

4. Ark Nova en medio del paisaje japonés 
Y en la línea de la utopía hay que nombrar al arquitecto argentino Tomás Saraceno $^{13}$, creador de estructuras hinchables flotantes como Poetic Cosmos of the Breath (2007), una instalación realizada en Reino Unido compuesta por una cúpula de 60 metros de diámetro cuyo funcionamiento dependía del Sol, ya que el calor de éste la ayudaba a inflarse [Fig. 5]. Esta estructura jugaba con la luz y el propio aire, creando una sensación inmersiva y psicodélica para quien quisiera habitarla.

Como los ejemplos mostrados, hay muchos otros con la arquitectura hinchable como protagonista; proyectos útiles que se alejan de la concepción tradicional y buscan innovar, como lo hicieron ya los arquitectos de los años 60. La arquitectura actual nos ha de dar cobijo, pero también debe cambiar y moverse a nuestro ritmo. Estas obras son un ejemplo de cómo existen alternativas a lo común, y que resultan igualmente válidas.

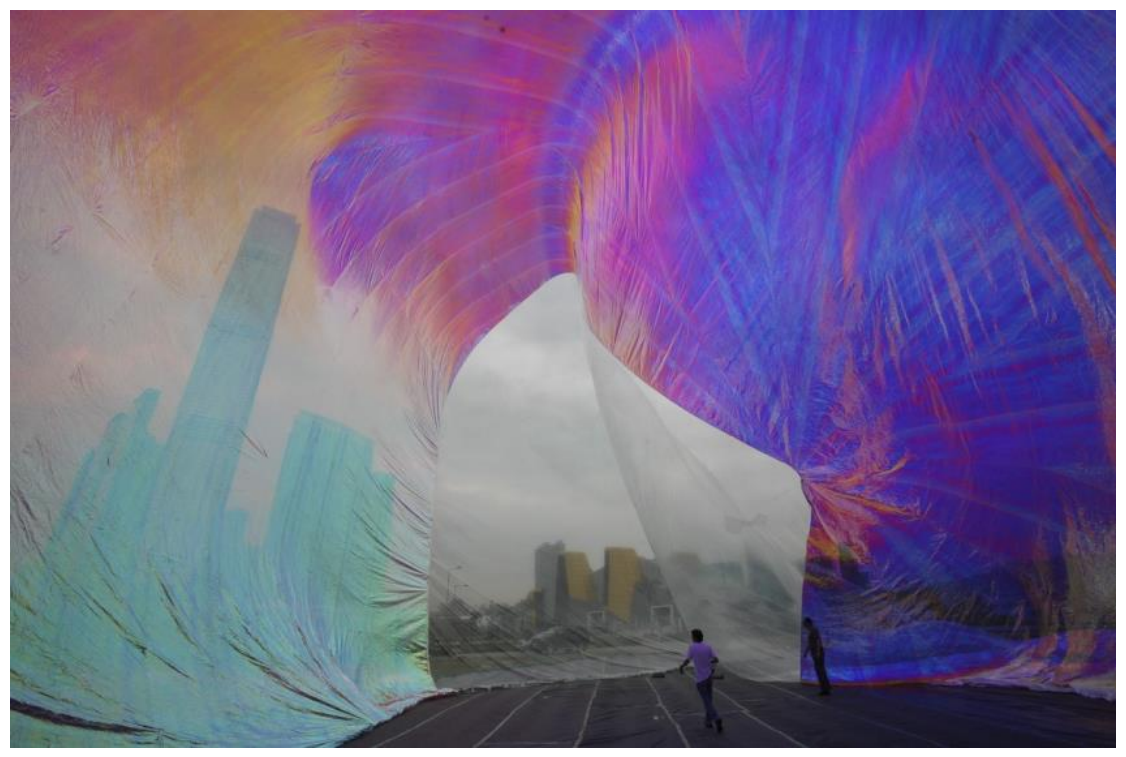

5. Obra de Tomás Saraceno

\section{CONCLUSIONES}

Tras haber realizado un análisis por la evolución y estado actual de la arquitectura efímera hinchable, se llega a la conclusión de lo necesarias que son para entender la habitabilidad y la concepción de los espacios en la actualidad. El aire se mueve, cambia, y las construcciones arquitectónicas cambian con ello. El ser humano muta y evoluciona. Las

${ }^{13}$ Web: https://studiotomassaraceno.org/ 
creaciones hinchables podrían ser una metáfora de nosotros mismos; necesitan el aire para vivir, para mantenerse en pie. Asimismo, su condición efímera las lleva a ser creaciones especiales que revolucionan sus espacios por periodos cortos, pero que permanecen en nuestras mentes si las hemos vivido; ejemplo de ello sería la Instant City de José Miguel de Prada Poole, aún recordada hoy en día por todo lo que supuso su creación ${ }^{14}$.

Todas estas creaciones presentadas en el artículo hacen ver, igualmente, lo funcionales que resultan las estructuras efímeras hinchables. A pesar de ser construidas con materiales baratos como el PVC y de tener un proceso de creación relativamente rápido, pueden suplir las mismas necesidades -a corto plazo- que las obras arquitectónicas tradicionales.

Lo que es cierto es que estas arquitecturas hinchables no han sido olvidadas. Son muchos los arquitectos que en todo el mundo siguen arriesgándose y pensando en hacer realidad las utopías. Y lo acaban consiguiendo.

\section{REFERENCIAS BIBLIOGRÁFICAS}

Baraona Pohl, E. (2009). “Ant Farm". Plataforma Arquitectura. Recuperado de https://www.plataformaarquitectura.cl/cl/02-18400/ant-farm. (Consulta: 10/02/2020)

Blasco Rodríguez, Carmen (2017). Hinchables. La Galería. Arquitectura, Arte y Diseño español. Conarquitectura: Madrid.

Movimiento Moderno. Hisour. Recuperado de https://www.hisour.com/es/modernmovement-33608/. (Consulta: 10/02/2020)

Prieto González, Nuria (2013). La arquitectura de José Miguel de Prada Poole: teoría y obra. Tesis Doctoral UDC. Universidad de La Coruña.

Ribadeneira, A. (2011). "El Manifiesto de Doorn Team 10”. Arquiteorías. Recuperado de https://arquiteorias.blogspot.com/2011/05/el-manifiesto-de-doorn-team-10.html. (Consulta: 07/02/2020)

${ }_{14}$ En los últimos años se han realizado diversas exposiciones retrospectivas sobre el arquitecto José Miguel de Prada Poole por sus grandes aportaciones al campo de la arquitectura y por ser pionero en la realización de arquitectura hinchable en España. 
Torrijos, P. (2013). "Construir castillos con el aire: la Ciudad Instantánea de Ibiza". JotDown. Recuperado de https://www.jotdown.es/2013/08/construir-castillos-con-elaire-la-ciudad-instantanea-de-ibiza/. (Consulta: 16/02/2020) 